\title{
Gestión moderna del mantenimiento de equipos pesados
}

\author{
Modern Heavy Equipment Maintenance Management
}

\author{
Manuel Enrique Zegarra Ventura ${ }^{1}$
}

http://dx.doi.org/10.21503/CienciayDesarrollo.2015.v18i1.05

\section{RESUMEN}

La gestión de maquinaria y equipos de construcción es una tarea difícil. Los responsables del equipo mecánico deben tomar decisiones técnicas y económicas complejas sobre el destino de las máquinas a su cargo. Estas decisiones incluyen temas relativos a adquisiciones, mantenimiento, reparaciones, reconstrucciones, eficiencias, costos, pronosticar las tasas de arrendamiento interno, disponibilidad, confiabilidad, reemplazos y bajas de activos. Las empresas constructoras de nivel internacional están inmersas en lo que se denomina El Mantenimiento de Clase Mundial, que significa tener políticas para realizar las labores cotidianas de trabajo con buenas prácticas tanto en el campo administrativo como en el campo técnico. Dentro del mantenimiento de los equipos, existen dos indicadores muy evaluados, que son la Disponibilidad Mecánica (DM) y la Confiabilidad (R). En este trabajo tratamos temas relativos a las buenas prácticas del mantenimiento de equipos, así como el significado y forma de avaluar los indicadores mencionados.

Palabras clave: mantenimiento, monitoreo de condiciones, disponibilidad mecánica, confiabilidad.

\section{ABSTRACT}

Machinery and Building Equipment Management is a difficult task. Those responsible for the mechanical machine must make complex technical and economic decisions on the fate of the machines in charge. These decisions include issues related to procurement, maintenance, repairs, reconstructions, efficiencies, costs, forecast internal lease rates, availability, reliability, and low asset replacements. Construction companies worldwide are engaged in what is called World Class Maintenance, which means having policies to perform everyday tasks working with best practices both in the administrative and in the technical field. Within the maintenance of equipment, there are two very evaluated indicators, which are the Availability Mechanics (DM) and Reliability (R). In this paper we address issues related to the good practices of maintenance equipment, as well as the meaning and how to assess the mentioned indicators.

Key words: maintenance, condition monitoring, availability mechanics, reliability.

1 Doctor en Administración de la UAP, Maestro en Administración de la USMP, Ingeniero Mecánico de la PUCP, CIP 49526, Diplomado en ingeniería mecatrónica por la URP, especialista CAD-CAE por la PUCP, Black Belt Six Sigma por Caterpillar. Trabajo actual: Jefe de Monitoreo y Planeamiento en la gerencia de Gestión de Equipos en la empresa Ferreyros, asesor para empresas constructoras, docente a tiempo parcial del curso Gestión de Mantenimiento de Equipo Pesado en la PUCP. Correo electrónico personal:mzegarraventura@hotmail.com;mzegarraventura@gmail.com 


\section{BASES TEÓRICAS SOBRE GESTIÓN DE EQUIPOS}

Existe documentación basta sobre teorías de la gestión de mantenimiento, sus procedimientos, objetivos y beneficios. Vamos a exponer algunas de ellas, que se consideran útiles para el enfoque que se requiere para el presente trabajo. "El máximo rendimiento de una pieza de equipo de minería depende primordialmente de tres factores críticos: el diseño del producto, la aplicación en que es usado, y el mantenimiento que ésta recibe durante su vida de servicio. En algún grado estos factores pueden ser controlados, pero algunos mucho más que otros." (Caterpillar, 2005, pág. 2). Este pensamiento puede aplicarse también a todo equipo de construcción, pues las máquinas y el trabajo realizado son muy similares. Las exigencias en el campo minero son mayores por el tipo de material y volúmenes que mueven, pero los principios del cuidado de las máquinas y los resultados que ello brinda son los mismos. Comenzaremos citando dos definiciones de mantenimiento, que creo reúne la esencia del pensamiento del mismo. "El mantenimiento es un conjunto de actividades técnicas, de aplicación directa, estructurales y de control económico que tiene como objetivo conseguir que la vida útil de las instalaciones, máquinas y edificios sea la mayor posible, lo que permite que el valor de las inversiones permanezca activo durante el tiempo de amortización e inclusive después “ (Valdivia, 1993, pág. 4). "Cuando hablamos de equipos móviles, el mantenimiento preventivo puede ser definido como una actividad organizada cuyo objetivo es maximizar el servicio y valor económico de la máquina. Estas actividades comienzan con la adquisición de la máquina y continúa a lo largo de su vida...." (Equipment Maintenance Council, 2007, pág. 117). Vemos pues, que la idea principal es mantener los activos en condición productiva, pero a la vez nos dice que el valor de las inversiones debe tener un control económico, para de esta manera maximi- zar utilidades. Esto implica que los sistemas de mantenimiento deberán tener una metodología y deberán servirse de herramientas adecuadas, para el logro de su objetivo. En este sentido podemos citar lo siguiente:

"El reto de manejar altos costos, intensivos en capital, equipos móviles, está siendo lograda, incorporando nuevos sistemas y tecnologías emergentes. Estas tecnologías están contribuyendo a tener una mejor y directa utilización de cada activo durante su vida útil y está poniendo énfasis en el manejo de los costos de operación y en el mantenimiento preventivo"... "Sin un adecuado sistema de monitoreo es imposible capturar la información necesaria, para manejarla efectivamente en una unidad. De acuerdo con el viejo axioma 'Si no puedes medirlo, no podrás manejarlo', es importante hacer un seguimiento y actividades de los equipos así como calificar y categorizar su uso" (Kruse, 1998).

La constante búsqueda de la eficiencia y competitividad enfrenta a la industria moderna a mayores y nuevos desafíos. La construcción no está ajena a este desafío y constantemente debe desarrollar, perfeccionar y modernizar, sus procesos constructivos, involucrando en estos cambios a sus equipos y personal. Actualmente, los equipos son usados en aplicaciones de mayor severidad, por lo que los fabricantes de equipos responden a estos cambios, incorporando en sus diseños avances tecnológicos en el área de la productividad, operación y control de estos equipos. El personal (operadores, técnicos, ingenieros, administrativos), se enfrentan a una constante actualización para trabajar con equipos de avanzada tecnología en este ambiente de alta exigencia.

Equipos más eficientes y de mayor tamaño y capacidad, así como la incorporación de la electrónica y computación en sus sistemas y mecanismos, son solo algunos ejemplos de estos cambios. En este ambiente de alta demanda, la gestión de 
equipos tiene un papel fundamental y cada vez más importante, para el logro de los objetivos finales, que son costos bajos de operación y alta productividad.

"La experiencia indica que existen funciones fundamentales a considerar en la estructura de un Sistema de Mantenimiento. Ellas son:

- Mantenimiento preventivo

- Monitoreo de condiciones

- Administración de reparaciones pendientes

- Administración de componentes

- Servicios de reparaciones en el taller y en el campo

- Registro del historial de reparaciones

- Análisis de resultados de gestión

- Administración de problemas.

Estas ocho funciones respaldan una gestión exitosa de mantenimiento" (Flores A., 1998).

Igual punto de vista tenemos en las expresiones del Ing. Fernando Harmsem, exdirector de la empresa Graña y Montero: "En un ambiente competitivo, con equipos de alto nivel tecnológico, es necesario perfeccionar los métodos de cómo ejecutar el mantenimiento en forma eficaz.

Calificar dentro de lo llamado MANTENIMIENTO CLASE MUNDIAL, significa estar organizado, para combatir los costos de la ineficiencia, convirtiéndonos en una valiosa contribución a la producción de la empresa. Las áreas que constituyen la base de la excelencia del mantenimiento son:

- Aspectos organizacionales

- Entrenamiento

- Órdenes de trabajo

- Planeamiento y programación

- Mantenimiento preventivo

- Compras y stock
- Información gerencial

- Automatización del mantenimiento.

Esto nos permitirá pasar de un mantenimiento de emergencia a un mantenimiento planificado, nos permite lograr reducciones del costo de mantenimiento e indisponibilidad, reducción de paradas inesperadas, reducción del tiempo de las reparaciones, aumento de la vida útil, aumento del valor de venta, aumento de la producción e incremento de las utilidades, apoyo efectivo a la elaboración de los presupuestos." (Harsem, 1993).

Las características del responsable de equipos, son claramente indicadas en el siguiente artículo: "La eficiencia y efectividad de la flota en su organización es responsabilidad exclusiva de una persona: usted. (refiriéndose al gerente de equipos). Por lo tanto las habilidades necesarias para la gerencia de flotas, involucran más que solo el cambio de aceite y reparaciones de motores. El gerente de equipos de hoy debe ser competente en recursos humanos, tecnología, costeo del ciclo de vida, negociación, gerencia de repuestos e instalaciones, benchmarking y seguridad y medio ambiente, para mencionar unos pocos. Y todas esas competencias contribuyen a la organización de su compañía, ahora más que nunca." (Equipment Maintenance Council, 2000).

Lo anterior, entonces, sugiere que el perfil de los responsables de los equipos de las empresas constructoras sean profesionales en ingeniería mecánica y además posean estudios de maestrías en administración de empresas con conocimientos de finanzas, ya que además de ser un profesional con una sólida base técnica, debe tener base administrativa con una visión diferente de la gestión de mantenimiento. Esto implica tener conocimientos sobre administración de personal, leyes laborales, procedimientos, organización, programación de operaciones, etc. Se debe dar resultados de gestión y se debe saber realizar análisis económicos de inversión en equipos y 
maquinarias. "Los gerentes de mantenimiento que tienen un control preciso del manejo de los reportes, seguimientos, y conocimiento de los costos totales reales, de sus operaciones son personas valiosas, en su compañía, u organización. El poder de esta información, y de este conocimiento, puede ser usado para el planeamiento financiero no solo de su sección sino de su organización. Estos costos de flota son solo parte de la foto integral de la organización. Si un gerente de flota no conoce estos costos, alguien más usará algunos, parte o tal vez información incorrecta, para hacer decisiones por usted. Su trabajo como gerente de flota o de equipos es generar la información correcta, usando métodos de recolección consistentes, defenderlos y hacer recomendaciones, basado en la data correcta. Esto ayudará a que la gerencia no malinterprete los costos de operación y tome decisiones en la empresa...." (Hinderaker, 1998).

"Si la organización es estructurada de tal forma que cada problema que impacta en el rendimiento de los equipos sea conocido, cuantificado, y comunicado a través de la organización, el esfuerzo en el mantenimiento (realmente la gestión de mantenimiento) puede ser extremada- mente efectivo al manejar sus problemas o mejor aún en evitarlos." (Caterpillar, 2005, pág. 3).

Estas definiciones y pensamientos de personas dedicadas al mantenimiento, con años de ejercicio profesional, coinciden todos en que el manejo de equipos, implica no solo el realizar mantenimientos, abastecimientos de combustibles, cambios de aceites, o reparaciones, sino realizar estas actividades con perspectiva económica y de conocimiento de los costos involucrados, no solo para conocerlos sino para controlarlos y manejarlos.

"Lo siguiente es verdadero para la mayoría de actividades incluyendo el mantenimiento:

- No se puede manejar lo que no se puede controlar.

- No se puede controlar lo que no se puede medir.

- No se puede medir sin una meta.

- Sin una meta no se puede mejorar" (Caterpillar, 2005, pág. 3).

Vemos pues que en la visión moderna del mantenimiento y de su gestión, también se hace énfasis en la planeación, control, mejoras, capacitación,

\section{Característica}

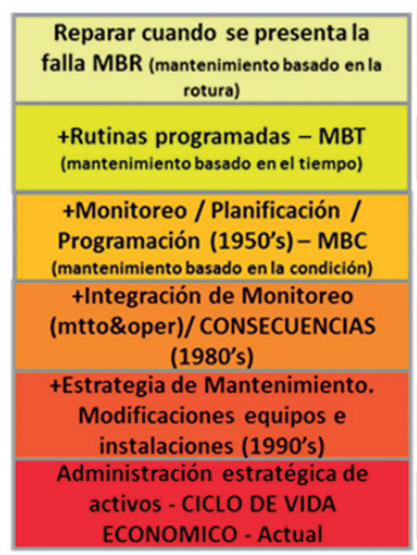

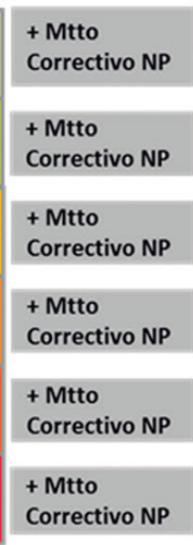
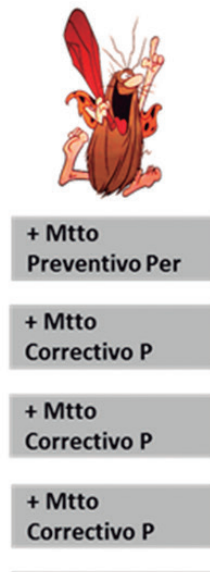

Correctivo P

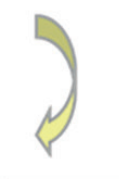

+ Mtto

Preventivo Per

+ Mtto

Preventivo Per

+ Mtto

Preventivo Per

Gerencia estratégica de Activos (SAM)

Figura 1. Evolución del mantenimiento (modificado por el autor) 
procedimientos, tal como sucede en la teoría de la administración.

Para analizar y realizar la mejora continua de cualquier proceso, es necesario conocer el tema técnico, las labores que en ellos se realiza, los objetivos que se persiguen. Un cambio en un proceso administrativo, puede afectar un parámetro técnico. Un sistema administrativo y un eficiente flujo de la información, no son la solución per sé de los problemas que se presenten en la gestión del mantenimiento de equipos. Una adecuada organización y un adecuado flujo de la información, nos permitirá conocer a través de los datos e información, a tiempo, cuándo existe un problema y nos permitirá actuar de forma más eficiente. Muchas veces la solución es completamente técnica. Otras veces la solución puede ser administrativa. Es por esta razón que consideramos que el responsable de la gestión de equipos no puede ser solo administrador. Debe poseer sólidas bases en ingeniería y contar con una gran perspectiva en gestión administrativa.

Es por esta razón que explicaremos algunos conceptos técnicos de ingeniería, inherentes a la gestión de mantenimiento, a fin de que se tenga una idea más amplia del contexto y de los parámetros a analizar a fin de realizar un mantenimiento de calidad. De esta manera se comprenderá la necesidad de tener una adecuada gestión del mantenimiento y de la importancia de tener una política de mejora continua.

\section{CONCEPTOS TÉCNICOS SOBRE MANTENIMIENTO MECÁNICO}

Las labores de mantenimiento se dividen en dos grandes grupos:

- Mantenimientos programados

- Mantenimientos no programados

Dentro de los mantenimientos programados, tenemos:
- Mantenimientos preventivos

- Mantenimientos predictivos

- Mantenimientos correctivos previstos o backlogs

Dentro de los mantenimientos no programados, tenemos:

- Mantenimientos correctivos imprevistos

La diferencia entre los mantenimientos correctivos previstos y los imprevistos es que los primeros son consecuencia de una inspección o evaluación previa, realizada en cualquiera de los mantenimientos programados. Los segundos ocurren inesperadamente y son mucho más costosos, porque incluyen piezas que no son de cambio normal además de venir acompañados de costos ocultos a causa de paralizaciones en la producción.

\section{MANTENIMIENTO PREVENTIVO}

Consiste en realizar las labores de engrase, lubricación y reparaciones menores o mayores programadas (antes de que ocurran las fallas), indicadas en las pautas y/o manuales de mantenimiento y lubricación, entregados por los fabricantes cuando se compra una máquina.

\section{MANTENIMIENTO PREDICTIVO}

Consiste en realizar evaluaciones programadas, a fin de obtener parámetros de funcionamiento de las máquinas y compararlos con los parámetros dados por los fabricantes. Incluye inspecciones visuales y auditivas, a fin de descubrir ruidos o señas fuera de lo esperado.

\section{MANTENIMIENTO CORRECTIVO}

Consiste en realizar labores de reparaciones luego que se ha presentado la falla. Es un tipo de actividad que puede ser programada (backlogs) 
o no programada. Si el mantenimiento es programado ha venido de alguna inspección de monitoreo de condiciones o de un servicio de mantenimiento preventivo o predictivo, por lo que va a significar que se hará una paralización programada y coordinada con el área de obras. No ocasionará mayor gasto que el mínimo necesario y previsto, pero si es no programado va a significar una parada inesperada de máquina, con las consecuencias inherentes, como son el retraso de la obra, costo de reemplazos de máquinas más caros, pago al personal administrativo con la obra paralizada, repercusión en otros frentes de obra, que tendrán que sufrir paralización también inesperada. Los mantenimientos correctivos programados requieren de menos repuestos que los no programados. Normalmente las reparaciones no programadas involucran cambios de repuestos que normalmente no deben ser cambiados nunca, o con muy poca frecuencia.

\section{CONTROL DE LA CONTAMINACIÓN}

Debido al desarrollo tecnológico y las exigencias y esfuerzos a las que las máquinas son sometidas, los fabricantes diseñan máquinas con tolerancias más precisas entre las piezas. Las luces (espacios o huelgos) que hay entre piezas que tienen movimiento relativo entre sí son muy pequeñas. Como estas piezas tienen movimientos relativos, debe existir entre ellas un lubricante, que evite el contacto directo entre las piezas y evitar de esta manera desgastes debido al rozamiento o abrasión, entre otros.

\section{MONITOREO DE CONDICIONES}

La tecnología da un impulso a la evolución del concepto de mantenimiento, pues permite aprovechar información relacionada al diagnóstico de fallas. La electrónica y las comunicaciones digitales, radiales y satelitales permiten obtener y conocer datos de los parámetros más importantes del funcionamiento de las máquinas (con ayuda de sensores, transmisores y receptores), los que son enviados a los servidores de los fabricantes, para que luego, por medio de un software que sirve como inter-fase HMI (buman machine interfase), se pueda acceder a dicha información a fin de que sea usada para prevenir fallas de magnitud.

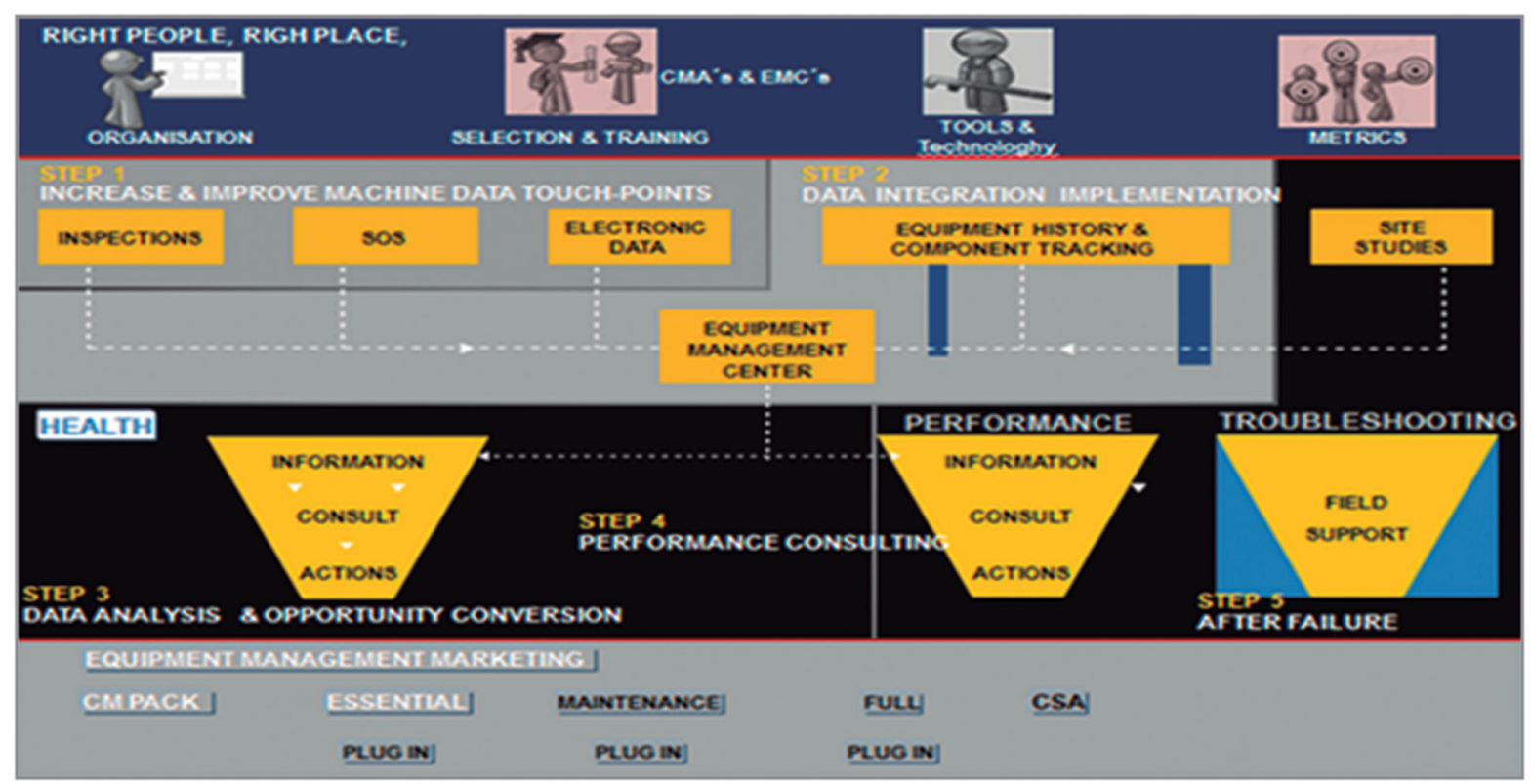

Figura 2. Los cinco elementos del monitoreo de condiciones (Caterpillar INC, 2011). 
El monitoreo de condiciones, adicionado a una evaluación de la planificación de la producción y de los ciclos de trabajo de las máquinas van a conducir al máximo rendimiento de estas en obra y por lo tanto a la maximización del buen uso de los recursos, lo que significa maximización de la productividad, con los beneficios económicos que ello trae.

\section{MAXIMIZACIÓN DE LA PRODUCCIÓN PARA LA MEJOR RENTABILIDAD DE LOS EQUIPOS}

La maximización de la producción es el objeto de toda empresa para poder conseguir máxima rentabilidad. La maximización va a depender de un adecuado planeamiento de la producción, adecuada selección de los equipos para el trabajo a realizar, adecuada operación del equipo, un adecuado índice de utilización de los equipos, adecuada disponibilidad mecánica y alta confiabilidad.

Vamos a tocar los dos últimos factores, por ser los más usados al momento de evaluar una gestión del mantenimiento.

\section{DISPONIBILIDAD MECÁNICA}

Es una relación directa de las horas producidas entre las horas producidas más las horas que la máquina estuvo en reparación. Es una relación entre el tiempo que la máquina produce y el tiempo que está en reparación. No confundir con el tiempo que la máquina podría estar produciendo, que ese es un indicador llamado uso de máquina. La fórmula del cálculo de la disponibilidad mecánica es como sigue:

$$
D M=\frac{\text { Horas Trabajadas }}{\text { Horas Trabajadas }+ \text { Horas en Reparación }}
$$

Si dividimos al numerador y al denominador de esta ecuación entre el número de reparaciones que se llevaron a cabo en el período de estudio:

$$
D M=\frac{\frac{\text { Horas trabajadas }}{\text { Número deparadas }}}{\frac{\text { Horas trabajadas }}{\text { Número de paradas }}+\frac{\text { Horas en reparación }}{\text { Número de paradas }}}
$$

Tenemos:

$$
\mathrm{DM}=\frac{\mathrm{TPEP}}{\mathrm{TPEP}+\mathrm{TPPR}}=\frac{\mathrm{MTBS}}{\mathrm{MTBS}+\mathrm{TPPR}}
$$

El TPEP o tiempo promedio entre paradas (llamado también MTBS o Mean Time Between Shutdowns) es un indicador que muestra el tiempo que la máquina trabaja en promedio antes de que suceda una reparación o parada. Incluye todo tipo de paradas de máquina como: mantenimientos programados, mantenimientos no programados, mantenimientos preventivos, mantenimientos correctivos. El TPPR o tiempo promedio por reparación (llamado también MTTR o Mean Time To Repair) es un indicador que muestra el tiempo promedio que demoran las reparaciones en la máquina. De igual manera, podemos o no considerar los tiempos de mantenimientos programados para cambios de aceite. Entonces podemos ver que para diferentes combinaciones de TPPR y TPEP podríamos tener la misma disponibilidad mecánica. Por esta razón es que la DM o disponibilidad mecánica debe analizarse con estos indicadores y no solo con la simpleza de saber las horas de trabajo y las horas en reparación. Un valor alto de TPEP nos dará indicativo de que la máquina falla poco, o tiene una frecuencia de reparaciones baja. Por el contrario, un valor bajo de este indicador nos dará la alerta que la máquina tiene una alta frecuencia de reparaciones. Un valor alto de TPPR nos estará indicando que demoramos mucho en efectuar las reparaciones. Por el contrario, un bajo 
valor de este indicador, nos dirá que tenemos tiempos bajos en promedio para las reparaciones en la máquina. Hay que tener cuidado de que un valor muy bajo en este indicador también podría significar que no hacemos las reparaciones siguiendo los procedimientos adecuados. En suma hay que observar los dos indicadores, para saber por ejemplo si un muy bajo TPPR está relacionado a un bajo TPEP. En otras palabras, una alta frecuencia en las reparaciones puede ser consecuencia de hacer las reparaciones demasiado apresuradas sin tomar el tiempo necesario y adecuado para ellas. Entonces podemos decir que un buen valor de TPEP es indicativo de una buena calidad de las reparaciones y un buen valor de TPPR es indicativo de un buen rendimiento de la mano de obra. Ambos indicadores nos alertarán si hay una falla al seguir los procedimientos y las buenas prácticas de mantenimiento y de taller.

\section{CONFIABILIDAD}

Es la probabilidad de que un equipo cumpla satisfactoriamente ( $\sin$ fallas) sus funciones definidas durante un período específico bajo condiciones de operación específica (Barlow, 1998, pág. xv).

"La distribución de Weibull es muy útil debido a que es la más inclusiva de todas las distribuciones y puede indicar qué sección de la curva en forma de tina de baño es sobresaliente. Las distribuciones de Weibull son mejores para el manejo de datos que pueden tener un número de características únicas y conforme a distribuciones diferentes en tiempos diferentes. La versatilidad de la distribución Weibull permite adecuar cada una de las diferentes distribuciones explicadas en la estadística, en una distribución utilizable. Esta distribución puede usarse para descifrar muchos tipos de problemas y es muy adecuada cuando se trata de fenómenos de la vida útil de los componentes y piezas" (Caterpillar, 2006, pág. 19).

\section{Distribución Weibull}

- Función de densidad acumulativa (CDF)

$$
F(x)=1-\exp \left[-\left(\frac{x-x_{0}}{\theta}\right)^{\beta}\right], \quad x \geq x_{0}
$$

- Función de densidad de probabilidad (PDF)

$$
f(x)=\frac{\beta\left(x-x_{0}\right)^{\beta-1}}{\theta^{\beta}} \exp \left[-\left(\frac{x-x_{0}}{\theta}\right)^{\beta}\right], \quad x \geq x_{0}
$$

Figura 3. Ecuación de Weibull con tres parámetros para la densidad acumulada de fallas (Caterpillar, 2006).

\section{Weibull Distribution}

\footnotetext{
- Has ability to fit different distributions, i.e., Normal, Lognormal and others

- $\beta=1$ : identical to the exponental distribution

$-\beta=2$ : identical to the Rayleigh distribution

$-\beta=2.5$ : approximates the lognormal distribution

- $\beta$ = 3.6: approximates the normal distribution

$-\beta=5$ : approximates the peaked normal distribution
}

\section{Figura 4. Interpretación de los valores de la} variable Beta (Caterpillar, 2006).

El parámetro de localización $\mathrm{X}_{0}$ se usa solo cuando la vida útil del producto comienza en algún número diseñado de horas de operación tales como los datos relacionados con la fatiga. No se usa cuando el punto de inicio es cero y simplifica en gran medida el uso de la distribución Weibull. El parámetro de forma $\beta$ describe la forma de la distribución y a su vez indica el tipo de problemas inherentes con la población.

- $\beta$ menor que 1 nos indica que hay una tasa de fallas decreciente.

- $\quad \beta$ igual a 1 indica que hay una tasa de fallas constante.

- $\quad \beta$ mayor a 1 indica que hay una tasa de fallas en aumento. 


\section{General Interpretation Guides for Beta $(\beta)$}

- Leak, loose bolts, manufacturing error, etc.

- Chance failures in useful lifer (normal operation) region

- Fatigue, pitting, spalling, or wear

- Wearout of systems

- Safety related components.

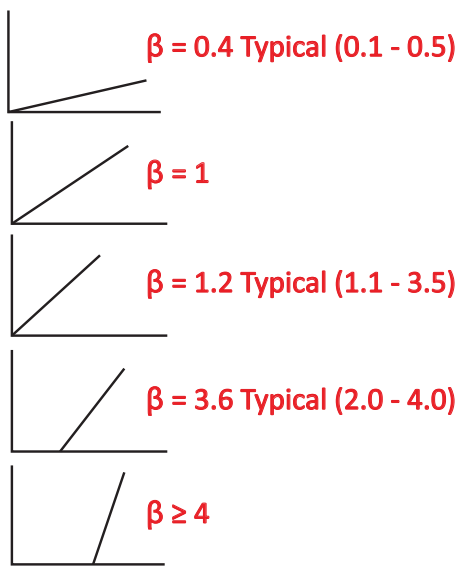

Figura 5. Interpretación aplicada de la variable Beta a la supervivencia (Caterpillar, 2006).

El parámetro de característica $\theta$ es la vida útil del $63,2 \%$ de la población que puede estar en términos del número de horas o millas o resistencia, etc. Es el punto pivote de la distribución y permanece así para cualquier valor o cambio de $\beta$. Este parámetro es análogo a la media en la distribución normal.
La tasa de falla está dada por la expresión:

$$
\text { Tasa de falla }=\lambda=\frac{\beta}{\theta^{\beta}}\left(x-x_{0}\right)^{\beta-1}
$$

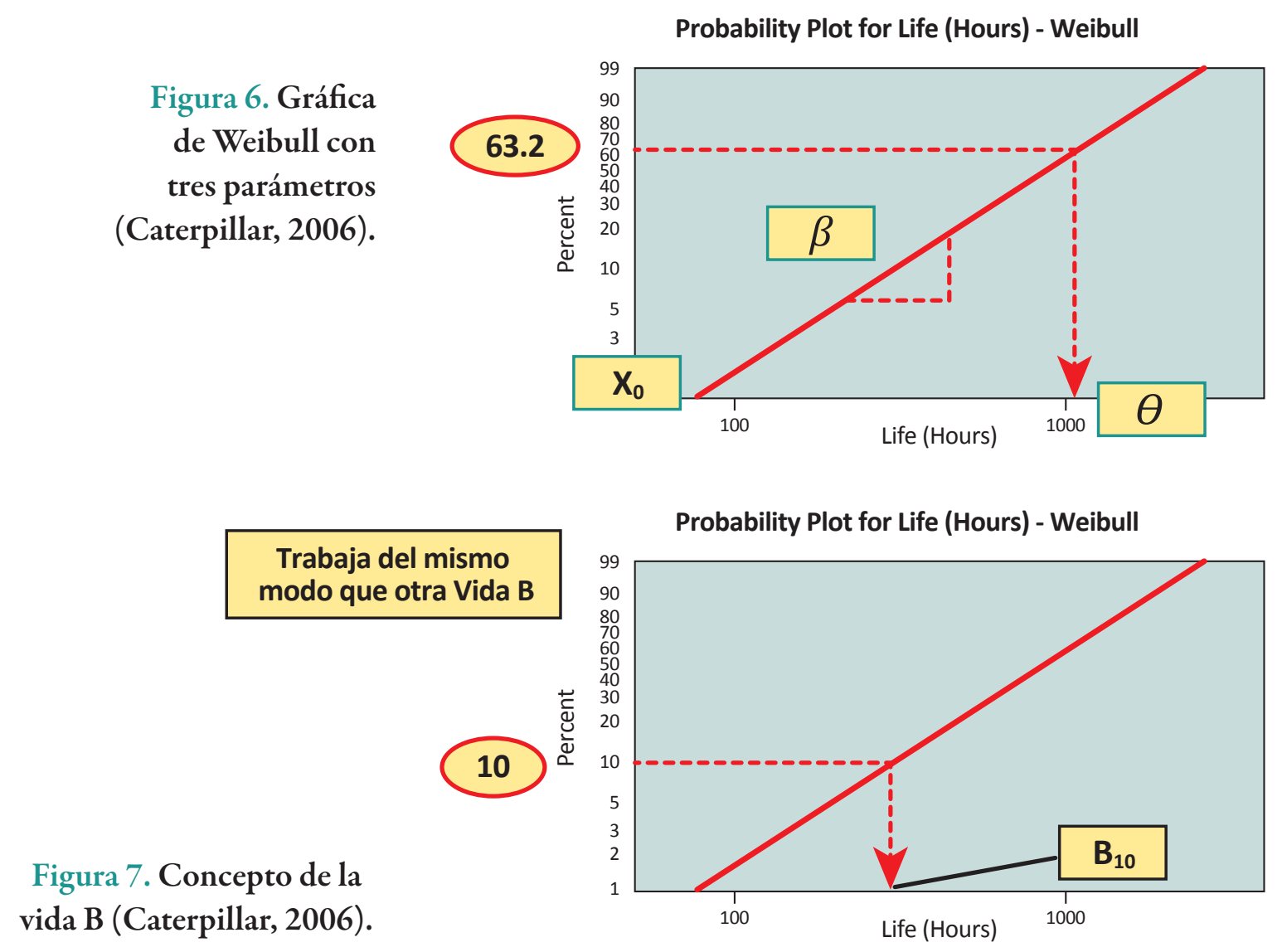




\section{Probability Plot for Life of CSA (Days) \\ Weibull $-70 \% \mathrm{Cr}$}

Censoring Column in CSA Status $(A=A c t i v e, C=$ Cancel $)$ - LSXY Estima tes

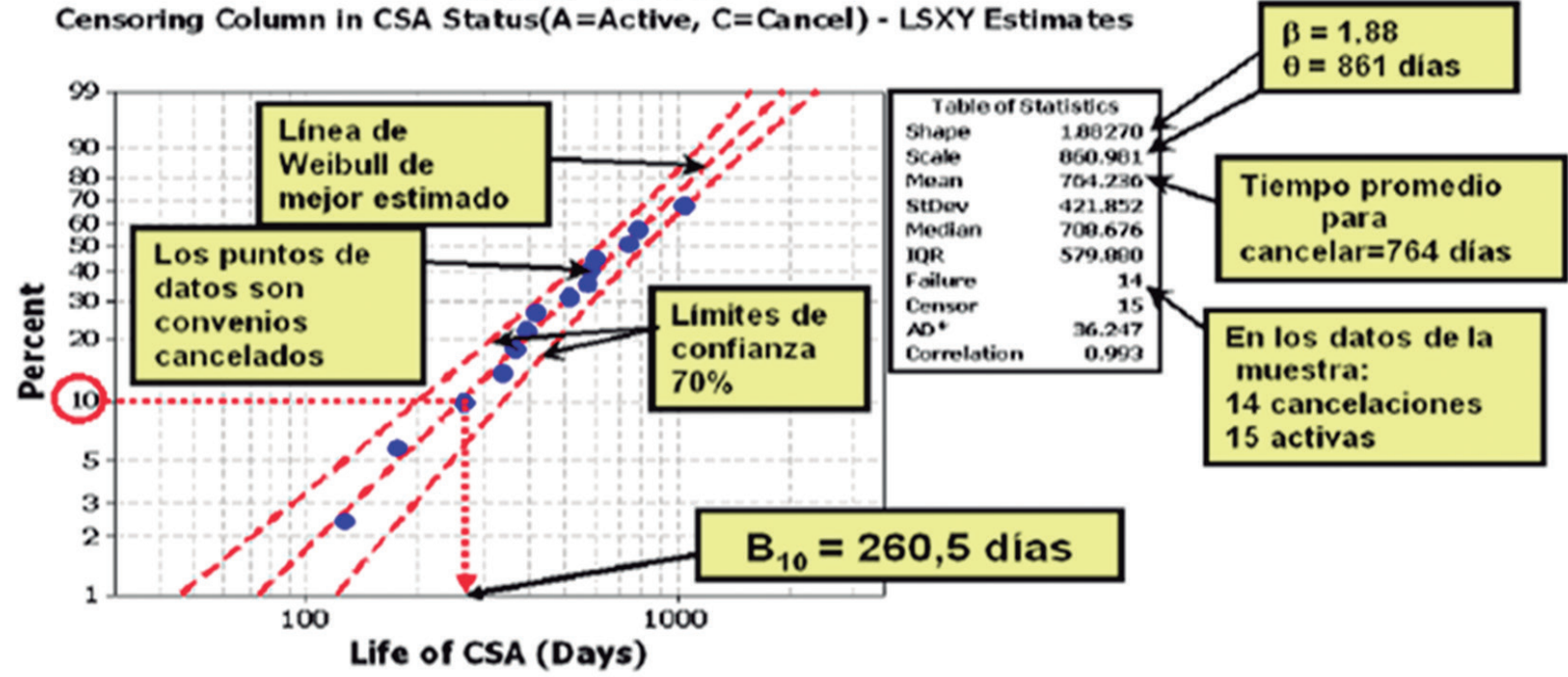

Figura 8. Ejemplo de significado de los parámetros Weibull (Caterpillar, 2006).

Expresado en términos de la distribución de Weibull con dos parámetros:

$$
R(t)=e^{(-t / \theta)^{\beta}}
$$

o

$$
R(t)=\exp ^{(-t / \theta)^{\beta}}
$$

Donde $\theta$ es el factor de escala o parámetro de característica y $\beta$ es el factor de forma.

Cuando la tasa de fallas es constante (algo que no es común), $\beta=1$.

La confiabilidad es normalmente expresada en términos de una ecuación exponencial, como:

$$
R(t)=e^{\left(-\frac{t}{\theta}\right)}=e^{\left(-\frac{t}{M T B F}\right)}
$$

o

$$
R(t)=e^{(-\lambda t)}
$$

Donde $\lambda$ es la tasa de fallas y MTBF (TPEF) es el tiempo promedio entre fallas.

$$
\lambda=1 / M T B F
$$

La mejora en la gestión, comparando dos períodos (antes y después), puede hacerse comparando los valores obtenidos ara cada período y luego haciendo una prueba de hipótesis para verificar si la diferencia en resultados es estadísticamente aceptable.

\section{REFERENCIAS}

1. Barlow, R. (1998). Engineering reliability. Philadelphia, USA: Sociaty for industrial and applied mathematics american statistical association.

2. Caterpillar. (2005). Performance metrics for mobile mining equipment ( $1 \mathrm{ed}$.). USA: Caterpillar.

3. Caterpillar. (2006). 6 Sigma, DMAIC (3.15 ed.). USA: Caterpillar.

4. Caterpillar. (2005). Performance metrics for mobile mining equipment ( $1 \mathrm{ed}$.). USA: Caterpillar.

5. Equipment Maintenance Council. (2000, mayo). Equipment Management Just Got Easier. Atlanta, Georgia, USA.

6. Equipment Maintenance Council. (2007). The career equipment fleet manager. 
7. Flores, A. (1998). El mantenimiento de equipo para la minería. USA: Caterpillar Americas.

8. Harsem, F. (1993). Mantenimiento clase mundial. Trujillo, La Libertad, Perú. Retrieved, 2013.

9. Hinderaker, P. (1998, Mayo). Acounting for Equipment Managers. Clear Water, Florida, USA: EMC Council.

10. Kruse, J. (1998). "The total mobile equipment management using satellite GPS and mobile data communications". In E. M. Council (Ed.). Clear Water - Florida.
11. Valdivia, G. (1993, agosto). La gestión de mantenimiento en la universidad. (APEMAN, Ed.) Mantenimiento. Retrieved, 2013.

12. Zegarra, M. La administración como herramienta de gestión para optimizar el mantenimiento mecánico en la empresa TyT SAC Contratistas Generales, año 2007. Tesis de Maestría, USMP, Lima, Perú.

13. Zegarra,M.Laaplicación demétodos administrativos para la mejora continua de la gestión de empresas constructoras medianas en Lima Metropolitana, año 2014. Tesis de doctorado, UAP, Lima, Perú. 